\title{
Decidual small extracellular vesicles induce trophoblast invasion by upregulating $\mathrm{N}$-cadherin
}

\author{
Mian Liu*, Xia Chen*, Qing-Xian Chang, Rui Hua, Yan-Xing Wei, Li-Ping Huang, Yi-xin Liao, \\ Xiao-Jing Yue, Hao-Yue Hu, Fei Sun, Si-Jia Jiang, Song Quan and Yan-Hong Yu \\ Department of Obstetrics and Gynecology, Nanfang Hospital, Southern Medical University, Guangzhou, China \\ Correspondence should be addressed to S Quan or Y-H Yu; Email: quansong@smu.edu.cn or yuyh1010@hotmail.com \\ *(M Liu and X Chen contributed equally to this work)
}

\begin{abstract}
Small extracellular vesicles (sEVs) are important mediators of cell-to-cell communication involved in the successful establishment of a pregnancy. Human decidual stromal cells play a key role in regulating trophoblast invasion. Nevertheless, the regulatory functions of decidual stromal cells-derived sEVs in human trophoblast cells are still unclear. In this study, primary human decidual stromal cells were isolated, and immortalized human endometrial stromal cell line (HESCs) were decidualized into human decidual stromal cells (HDSCs) using hormonal cocktail containing medroxy progesterone 17-acetate (MPA), estrogen and cAMP analog. HDSC-sEVs were isolated from both primary human decidual stromal cells and immortal HDSCs, respectively, and identified by transmission electron microscopy and western blotting. EV uptake assay indicated that HDSC-sEVs could be uptaken by trophoblast cells. HDSC-sEVs could increase the invasiveness and the expression level of $\mathrm{N}$-cadherin of trophoblast cells with elevated phosphorylation of SMAD2 and SMAD3 in the cells. Silencing of N-cadherin could block cell invasion induced by HDSC-sEVs, while knockdown of SMAD2 and SMAD3 could inhibit the upregulation of $\mathrm{N}$-cadherin in trophoblast cells. Taken together, our results suggested a regulatory effect of HDSC-sEVs in the invasion of trophoblast cells, and HDSC-sEVs may be important mediators of trophoblasts during embryo implantation and placentation.

Reproduction (2020) 159 171-180
\end{abstract}

\section{Introduction}

Successful pregnancy depends on the normal process of endometrial decidualization, embryo implantation, and placentation. Each step is indispensable to the next (Carson et al. 2000, Dey et al. 2004). Trophoblasts are specialized cells of placenta that play a major role in the implantation and formation of the maternal-fetal interface. Unlike uncontrolled invasion observed in tumors, trophoblastic invasion is a highly regulated process (Red-Horse et al. 2004). Inappropriate trophoblast invasion is implicated in several pregnancyrelated disorders, such as intrauterine growth restriction and preeclampsia (PE) (Brosens et al. 1967, Pijnenborg et al. 1991, Sankaralingam et al. 2006). It is important to unravel the molecular mechanisms in regulating trophoblast invasion.

Endometrium undergoes decidualization before the invasion of trophoblast. The significant change occurred during decidualization is the transformation of endometrial stromal cells into highly specialized decidual stromal cells that exhibit a distinct function (Gellersen and Brosens 2014, Sharma et al. 2016). The cross-talk between trophoblast and decidual stromal cells plays a key role in the appropriate invasion of trophoblast cells (Sharma et al. 2016). Decidual stromal cells could secrete many pro- and anti-invasion factors that may balance the invasiveness of trophoblast cells (Godbole et al. 2011). However, the mechanisms of how decidual stromal cells regulate trophoblastic invasion are not fully understood and further investigation may lead to a deeper understanding of the pathology of placenta-related complications.

Extracellular vesicles (EVs) have recently evoked considerable interest among researchers as these are released by many types of cells containing different cargoes, such as microRNA, mRNA and chemokines, which play important roles in cell-to-cell communication. Small EVs (sEVs) represent a particular subtype of extracellular vesicles with the diameter of 40-300 nm and were reported to be involved in regulating the activity of both neighboring and distant recipient cells through their signaling molecules (Maas et al. 2017, Théry et al. 2018). It has been reported that sEVs derived from either embryo or endometrium are involved in endometrial receptivity and may reflect placental health status (Homer et al. 2017, Tannetta et al. 2017). sEVs could promote the invasion of many 
types of cancer cells through epithelial-mesenchymal transition (EMT) by upregulating the expression level of $\mathrm{N}$-cadherin (Gopal et al. 2017). N-cadherin is one of the cell adhesion molecules that belongs to the cadherin subclasses and is a hallmark of EMT (Hansen et al. 2008, Mrozik et al. 2018). During implantation, trophoblast cells exhibit invasive ability akin to that of cancer cells and $\mathrm{N}$-cadherin is involved in this process (Zhao et al. 2018).

This study aimed to determine the role of sEVs derived from human decidual stromal cells in regulating trophoblast function and to identify the molecular mechanisms involved in this process. Our research demonstrated that human decidual stromal cellsderived sEVs (HDSC-sEVs) could induce invasiveness of trophoblast cells and that this effect was induced via the upregulation of $\mathrm{N}$-cadherin through SMAD2/3 signaling pathway. These findings might provide further evidence to support the key role of human decidual stromal cells in modulating the invasion of trophoblasts in a controlled manner required for a successful pregnancy.

\section{Materials and methods}

\section{Ethics}

Thirty first-trimester human placental specimens (gestational age: $6-12$ weeks) were obtained from women undergoing legal elective termination of pregnancy. This study was approved by Nanfang Hospital, Southern Medical University (Study number: NFEC-2017-055). All procedures were performed in accordance with the Declaration of Helsinki. Written informed consent was obtained from all pregnant women involved in this study.

\section{Tissue collection and isolation of primary trophoblast cells}

The methodology for the isolation of extravillous trophoblast (EVT) cells from first-trimester human placenta was described elsewhere (Li et al. 2018). Briefly, placental tissues (6-12 weeks, $n=15)$ were washed three times with cold sterile Dulbecco's PBS (Gibco, Invitrogen) and digested with trypsin (Gibco) and Dispase/DNAse (Gibco) with agitation at $300 \mathrm{rpm}$ for $20 \mathrm{~min}$ at $37^{\circ} \mathrm{C}$. The remaining tissues were sent for another three cycles of digestion. Fetal bovine serum $[20 \%(\mathrm{v} / \mathrm{v})(\mathrm{FBS}, \mathrm{PAN}-\mathrm{Biotech})]$ was added to terminate the digestion. All the tissues were collected and layered using Percoll (Gibco) gradient [20\% (v/v), 40\% (v/v), and $60 \%(\mathrm{v} / \mathrm{v})]$ centrifugation at $994 \mathrm{~g}$ for $20 \mathrm{~min}$ at $4{ }^{\circ} \mathrm{C}$. The primary trophoblast cells were collected from the $40 \%$ $(\mathrm{v} / \mathrm{v}) / 60 \%(\mathrm{v} / \mathrm{v})$ interfaces. Cells were resuspended in PBS and centrifuged at $300 \mathrm{~g}$ for $10 \mathrm{~min}$ at $4^{\circ} \mathrm{C}$. Subsequently, the cells were plated in tissue culture flasks in RPMI 1640 Medium (Gibco) with 20\% FBS, streptomycin (100 mg/ $\mathrm{mL})$ and penicillin $(100 \mathrm{U} / \mathrm{mL})$ at $37^{\circ} \mathrm{C}$ in a humidified $5 \%$ $\mathrm{CO}_{2}$ /air atmosphere.

\section{Immunofluorescence analyses of primary human EVTs}

Primary human EVTs $\left(1.5 \times 10^{4}\right)$ were seeded onto the $15 \mathrm{~mm}$ coverslips and allowed to attach for $24 \mathrm{~h}\left(37^{\circ} \mathrm{C}, 5 \% \mathrm{CO}_{2}\right)$ in RMPI 1640 with $20 \%$ FBS. Cells were then fixed with $4 \%$ paraformaldehyde for $10 \mathrm{~min}$ and washed twice with glutaraldehyde. Immunofluorescence staining was performed as previously described (Johnson et al. 2001). After being washed with PBS containing $0.3 \%$ vol/vol Tween 20, cells were blocked with $10 \% \mathrm{vol} / \mathrm{vol}$ goat serum and then incubated overnight at $4{ }^{\circ} \mathrm{C}$ with a MAB to human cytokeratin 7 (1 mg/ $\mathrm{mL}$, Abcam, AB9021). Cell-bound primary antibody was then detected using goat anti-mouse IgG Alexa $488(1 \mathrm{mg} / \mathrm{mL}$, Life Technologies). Coverslips were adhered to corning precleaned single-frosted slides via Prolong Antifade Mounting Reagent with DAPI (Invitrogen).

\section{Isolation of primary human DSCs and treatments}

Human decidua tissues were obtained following legal surgically terminated pregnancies at the Department of Obstetrics and Gynecology, Nanfang Hospital. Primary human decidual stromal cells were isolated according to the previously reported methods (Li et al. 2010). In brief, decidua tissues were obtained following elective terminations of pregnancy $(n=15)$, washed in PBS three times and minced into small pieces in phenol red-free DMEM/F12 in which tissues were digested with $0.1 \%$ collagenase and $0.1 \mathrm{mg} / \mathrm{mL}$ DNase for $1 \mathrm{~h}$ at $37^{\circ} \mathrm{C}$. The enzymatic reaction was terminated by adding phenol redfree DMEM/F12 medium with $20 \%$ (vol/vol) fetal calf serum (Gibco). The suspension was filtered through a sterile $100 \mu \mathrm{m}$ pore gauze mesh centrifuged at $400 \mathrm{~g}$ for $15 \mathrm{~min}$. Cell pellet was suspended in phenol red-free DMEM/F12 and centrifuged using a discontinuous Percoll (Sigma) gradient of 20, 40 and $60 \%$ ( $\mathrm{vol} / \mathrm{vol})$ at $994 \mathrm{~g}$ for $20 \mathrm{~min}$. The human DSCs were collected from the $20-40 \%$ interface and then suspended in phenol red-free DMEM/F12 medium with 20\% (vol/vol) FCS and incubated in cell plate. After cultured for $45 \mathrm{~min}$, the nonadherent lymphocytes were removed.

After the isolation of human DSCs, we cultured primary human DSCs in phenol red-free DMEM/F12 (Gibco) containing $10 \%(\mathrm{v} / \mathrm{v})$ charcoal-stripped fetal bovine serum (CS-FBS, PANBiotech, Bavaria, Germany), 3.1 g/L glucose (Sigma-Aldrich) and $1 \mathrm{mM}$ sodium pyruvate (Sigma-Aldrich) supplemented with $50 \mathrm{mg} / \mathrm{mL}$ penicillin-streptomycin (Gibco), $1.5 \mathrm{~g} / \mathrm{L}$ sodium bicarbonate (Sigma-Aldrich), $500 \mathrm{ng} / \mathrm{mL}$ puromycin (Sigma-Aldrich) and 1\% insulin-transferrin-selenium (Gibco). The primary human DSCs were passaged three to four times before in vitro treatments. We re-decidualized primary human DSCs with medium containing $1 \%$ CS-FBS, $10^{-8} \mathrm{M}$ estrogen $(\mathrm{E}$, Sigma-Aldrich) plus $10^{-7} \mathrm{M}$ medroxyprogesterone-17-acetate (MPA, Abcam) and $0.5 \mathrm{mM}$ dibutyryl cAMP (db-cAMP, SigmaAldrich) for 8 days as previously reported (James-Allan et al. 2018). Primary HDSCs were cultured using DSC culture media with $10 \%$ EVs-depleted FCS, $10^{-8} \mathrm{M}$ E plus $10^{-7} \mathrm{M}$ MPA and $0.5 \mathrm{mM} \mathrm{db}$-cAMP for $48 \mathrm{~h}$ and the conditioned medium was collected to isolate primary HDSC-sEVs. The EVs-depleted FCS was prepared as previously reported (Yan et al. 2018). In brief, we prepared EVs-depleted FSB by overnight ultracentrifugation of FCS at $100,000 \mathrm{~g}$ at $4{ }^{\circ} \mathrm{C}$. 


\section{Immunofluorescence analyses of primary human DSCs}

Primary human DSCs $\left(1.5 \times 10^{4}\right)$ were seeded onto the $15 \mathrm{~mm}$ coverslips and allowed to attach for $24 \mathrm{~h}\left(37^{\circ} \mathrm{C}, 5 \% \mathrm{CO}_{2}\right)$ in phenol red-free with $20 \%$ FCS. Cells were then fixed with $4 \%$ paraformaldehyde for $10 \mathrm{~min}$ and washed twice with glutaraldehyde. Immunofluorescence staining was performed as previously described (Johnson et al. 2001). After being washed using PBS containing $0.3 \%$ vol/vol Tween 20, cells were blocked with $10 \%$ ( $\mathrm{vol} / \mathrm{vol})$ goat serum followed by incubation overnight at $4{ }^{\circ} \mathrm{C}$ with a MAB to human vimentin ( 1 $\mathrm{mg} / \mathrm{mL}, \mathrm{Abcam}, \mathrm{AB} 193555)$ and a MAB to human cytokeratin 7 (1 mg/mL, Abcam, AB9021). Cell-bound primary antibody was then detected with goat anti-Rabbit IgG Alexa 594 (1 mg/ $\mathrm{mL}$, Life Technologies) and goat anti-mouse IgG Alexa 488 (1 $\mathrm{mg} / \mathrm{mL}$, Life Technologies). Coverslips were adhered to corning pre-cleaned single-frosted slides via Prolong Antifade Mounting Reagent with DAPI (Invitrogen).

\section{Immortalized HESCs culture and treatments}

The immortalized human endometrial stromal cell line (HESC) was generously provided by Prof. Haibin Wang (Xiamen University) and cultured according to the manufacturer's instructions (Krikun et al. 2004). HESCs were cultured in phenol red-free DMEM/F-12 (Gibco) containing 10\% (v/v) charcoal-stripped fetal bovine serum (CS-FBS, PAN-Biotech, Bavaria, Germany), $3.1 \mathrm{~g} / \mathrm{L}$ glucose (Sigma Aldrich) and $1 \mathrm{mM}$ sodium pyruvate (Sigma-Aldrich) supplemented with $50 \mathrm{mg} / \mathrm{mL}$ penicillin-streptomycin (Gibco), $1.5 \mathrm{~g} / \mathrm{L}$ sodium bicarbonate (Sigma-Aldrich), $500 \mathrm{ng} / \mathrm{mL}$ puromycin (SigmaAldrich) and $1 \%$ insulin-transferrin-selenium (Gibco).

The in vitro stromal cell decidualization was performed as described elsewhere (Szwarc et al. 2018). The endometrial stromal cells were treated with medium containing $1 \%$ CS-FBS, $10^{-8} \mathrm{ME}$ (Sigma-Aldrich) plus $10^{-7} \mathrm{MP}$ (medroxyprogesterone17-acetate, Abcam) and $0.5 \mathrm{mM}$ dibutyryl cAMP (SigmaAldrich). The media were changed every $48 \mathrm{~h}$, and the cultures were maintained for up to 8 days.

We cultured both types of HDSC by DSC culture media containing hormonal cocktail $\left(10^{-8} \mathrm{ME}\right.$ plus $10^{-7} \mathrm{M} \mathrm{MPA}$, and $0.5 \mathrm{mM}$ db-CAMP) with $10 \%$ EVs-depleted FCS for $48 \mathrm{~h}$ and collected the conditioned medium to isolate HDSC-sEVs.

\section{Immortalized extravillous trophoblast cell line HTR-8/SVneo and treatment}

The EVT cell line HTR-8/SVneo was purchased from American Type Culture Collection. The cell line is generated from the primary culture of cytotrophoblast cells obtained from explant cultures of human first-trimester placenta, as described elsewhere (Graham et al. 1993). HTR-8/SVneo cells were cultured in RPMI 1640 (Gibco) supplemented with 10\% FBS (PAN-Biotech) and $50 \mathrm{mg} / \mathrm{mL}$ penicillin-streptomycin.

For the experiments described, cells were seeded in six-well tissue culture plates until $60-80 \%$ confluence, cultured in
FBS-free media for 12-14 h and then treated with HDSC-sEVs $(25 \mu \mathrm{g} / \mathrm{mL}, 50 \mu \mathrm{g} / \mathrm{mL})$ for $48 \mathrm{~h}$.

\section{Isolation of HDSC-sEVS}

HDSC-sEVs were isolated from the cell culture supernatant as described elsewhere (Wu et al. 2018) with some modifications. In brief, the culture supernatant of human stromal cells was centrifuged at $300 \mathrm{~g}$ for $10 \mathrm{~min}, 2200 \mathrm{~g}$ for $30 \mathrm{~min}$ at $4^{\circ} \mathrm{C}$, and $10,000 \mathrm{~g}$ for $45 \mathrm{~min}$ at $4^{\circ} \mathrm{C}$ to eliminate cells and debris. The supernatant fluid was filtered using a $0.22 \mu \mathrm{m}$ sterile filter (Millipore, Billicera) and then ultracentrifuged at 100,000 $\mathrm{g}$ for $70 \mathrm{~min}$ at $4{ }^{\circ} \mathrm{C}$. The pellets were resuspended in a larger volume of PBS and ultracentrifuged at the same high speed for an additional $70 \mathrm{~min}$.

\section{Identification of HDSC-sEVs}

For transmission electron microscopy analysis, sEVs were fixed in $2 \%$ glutaraldehyde, planted onto carbon-coated grids and stained with phosphotungstic acid. Grids were examined using a transmission electron microscope $(\mathrm{H}-7650$, Hitachi High-Technologies, Tokyo, Japan). We tested sEVs classical markers such as Alix (1:1000, CST), CD81 (1:1000, Abcam), CD63 (1:1000, Abcam) and GAPDH (1:1000, CST). Western blotting was performed as previously described (Li et al. 2014).

\section{Fluorescence labeling of HDSC-sEVs}

HDSC-sEVs purified from cell cultured media were resuspended in diluent $\mathrm{C}$ from the lipophilic PKH-67 labeling kit (Sigma-Aldrich) and labeled according to the manufacturer's instructions. In brief, HDSC-sEVs were incubated in diluent C at $37^{\circ} \mathrm{C}$ for $10 \mathrm{~min}$. Excess dye was removed by washing with PBS and ultracentrifugation at $100,000 \mathrm{~g}$ for $70 \mathrm{~min}$ at $4{ }^{\circ} \mathrm{C}$. The labeled sEVs were pelleted by $\mathrm{PBS}$ and stored at $-80^{\circ} \mathrm{C}$ (maximum of 4 weeks) for downstream experiments, including uptake assays.

\section{Uptake of HDSC-sEVs by trophoblast cells}

HTR8/SVneo cells and primary human EVTs were plated on chamber slides, treated with a final concentration of $28 \mathrm{ng} / \mathrm{mL}$ labeled HDSC-sEVs (Green) for $4 \mathrm{~h}$, fixed with $4 \%$ paraformaldehyde at room temperature for $20 \mathrm{~min}$, permeabilized with ice-cold acetone at room temperature for $5 \mathrm{~min}$ and subsequently stained with DAPI (blue) as per the manufacturer's recommendations (Pantra, Guangzhou, China). Confocal images of trophoblast cells were acquired on a Zeiss Pascal system (Zeiss LSM 880). Z-stack images of HTR8/SVneo cells were recorded using a Z-step size of 0.5 $\mu \mathrm{m}$ and acquired on a Zeiss Pascal system. Image processing was performed using the ZEN 2 software (Zeiss), and 3D data sets were deconvolved using SoftWoRx (Applied Precision, GE Healthcare). For the analysis of cellular internalization of HDSC-sEVs, images were also deconvolved using ZEN 2 software. 


\section{Matrigel invasion assay of HTR8/SVneo cells treated with HDSC-sEVs}

The upper chamber of $8 \mu \mathrm{m}$ pore-sized Transwell inserts (Corning) was pre-coated with $60 \mu \mathrm{L}$ of pre-diluted Matrigel (Corning). The $2 \times 10^{4}$ pre-starved HTR8/SVneo cells were suspended in $250 \mu \mathrm{l}$ RPMI-1640 (Gibco) with HDSC-sEVs (25 $\mu \mathrm{g} / \mathrm{ml}, 50 \mu \mathrm{g} / \mathrm{ml}$ ) and $0.1 \%$ EVs-depleted FCS. Cell suspensions were seeded in upper chambers for $48 \mathrm{~h}$. RPMI1640 supplemented with $10 \%$ EVs-depleted FCS was added to the lower chamber as a chemoattractant. After incubation for $48 \mathrm{~h}$, the cells in the upper side of the insert were removed with a cotton swab and the cells on the lower side were fixed in absolute methanol for $20 \mathrm{~min}$ and stained with $0.1 \%$ crystal violet (Beyotime, Shanghai, China) for $30 \mathrm{~min}$. Five microscopic fields on the membrane of the chamber were examined under an Olympus IX 71 inverted microscope (Olympus) for counting the number of crystal violet-positive cells, which represent the invasive cells. Mean values from a minimum of five experiments were used in the analysis.

\section{Quantitative real-time polymerase chain reaction analysis}

Total RNA extracted from HTR8/SVneo cells were treated with HDSC-sEVs $(25 \mu \mathrm{g} / \mathrm{mL}, 50 \mu \mathrm{g} / \mathrm{mL})$, and primary trophoblasts were treated with primary HDSC-sEVs $(25 \mu \mathrm{g} / \mathrm{mL}, 50 \mu \mathrm{g} / \mathrm{mL})$ for $24 \mathrm{~h}$ and processed for RNA extraction. The transcript levels of $\mathrm{N}$-cadherin were assessed by real-time PCR, as described elsewhere (Li et al. 2014) The primers used for realtime PCR were $\mathrm{N}$-cadherin, 5'-GGA CAG TTC CTG AGG GAT CA-3' (forward) and 5'-GGA TTG CCT TCC ATG TCT GT-3' (reverse); human glyceraldehyde-3-phosphate dehydrogenase (GAPDH), 5'-GAG TCA ACG GAT TTG GTC GT-3' (forward) and 5'-GAC AAG CTT CCC GTT CTC AG-3' (reverse); SMAD2, 5'-GCC TTT ACA GCT TCT CTG AAC AA-3' (forward) and 5'-ATG TGG CAA TCC TTT TCG AT-3' (reverse); SMAD3, 5'CCC CAG CAC ATA ATA ACT TGG-3' (forward), and 5'-AGG AGA TGG AGC ACC AGA AG-3' (reverse). Expression levels were determined using the $2^{-\Delta \Delta C t}$ threshold cycle method. All experiments were performed five times.

\section{Western blotting}

Western blotting was performed as previously described (Li et al. 2014). The cells were washed three times in cold PBS and lysed in ice-cold lysis buffer (KeyGen Biotech, Nanjing, China). Total protein concentration was determined by BCA method (Thermo Fisher). Protein samples were loaded onto $10 \%$ (v/v) SDS-PAGE, resolved at $120 \mathrm{~V}$ for 70 min, transferred to nitrocellulose membranes (Millipore) and subsequently blocked with 5\% BSA (Sigma-Aldrich). After blocking, the membranes were incubated overnight at $4{ }^{\circ} \mathrm{C}$ with primary antibodies against $\mathrm{N}$-cadherin (1:1000, CST,MA,USA), (1:1000, CST), Phospho-SMAD2 (1:1000, CST), Phospho-SMAD3 (1:1000, CST), SMAD2 (1:1000, CST), SMAD3 (1:1000, CST), and GAPDH (1:1000, CST). The blots were washed and incubated with a secondary antibody conjugated with peroxidase (goat anti-Rabbit IgG, Santa
Cruz). Immunolabeling was performed using the ECL Western Blotting Substrate Pierce Kit (Thermo Scientific) in the Tanon 6200 Luminescent Imaging Workstation (Tanon, Shanghai, China). GAPDH was used as the loading control.

\section{Small interfering RNA transfection}

siRNAs for targeting N-Cadherin, SMAD2, SMAD3, and scrambled negative control siRNA were purchased from Guangzhou RiboBio Co. Ltd. Approximately 50\% confluent cells were transferred with $20 \mathrm{nM}$ siRNA and $20 \mathrm{nM}$ of its negative control by lipofectamine 2000 (Invitrogen), in accordance with the manufacturer's instructions. RT-qPCR and Western blotting analysis were performed to examine the transfection efficiency. Twenty-four hours post-transfection, the HTR-8/SVneo cells were treated with HDSC-sEVs $(25 \mu \mathrm{g} / \mathrm{mL})$.

\section{Statistical analysis}

All values were shown as means \pm SEM of at least five independent experiments. Nonparametric test was performed. Statistical evaluation of the data was performed by KruskalWallis test for comparisons among multiple groups and by the Mann-Whitney test for comparisons between two groups as appropriate. Statistical analysis was performed using SPSS19.0 program. The difference was regarded statistically significant if the two-tailed $P$-value was $<0.05$.

\section{Results \\ Isolation and identification of HDSC-sEVs in culture media}

We isolated the HDSC-sEVs from the culture medium of immortal human decidual stromal cells. The isolated sEVs were identified by electron microscopy and Western blotting. The electron microscopic image of typical sEVs is demonstrated in Fig. 1A. Meanwhile, the isolated sEVs were identified by the presence of endosomal markers, CD63, tetraspanin CD81, and Alix (Fig. 1B). We also isolated HDSC-sEVs from the culture media of primary human decidual stromal cells. The purity of isolated human DSCs was assessed by positive immunocytochemical staining of the fibroblast marker, vimentin, and negative expression of the trophoblast marker, CK7 (Supplementary Fig. 1A, see section on supplementary materials given at the end of this article). Immunocytochemistry has shown that vimentinpositive cells made up for above $95 \%$. Primary HDSCsEVs were identified by electron microscopy and Western blotting (Supplementary Fig. 1B and C).

\section{Uptake of HDSC-sEVs by trophoblast}

We hypothesized that HDSC-sEVs modulate the uterine microenvironment via a paracrine mechanism after their uptake by the adjoining cells. To determine the uptake of HDSC-sEVs by placental trophoblast cells, we investigated HDSC-sEVs uptake kinetics in HTR-8/ 
A
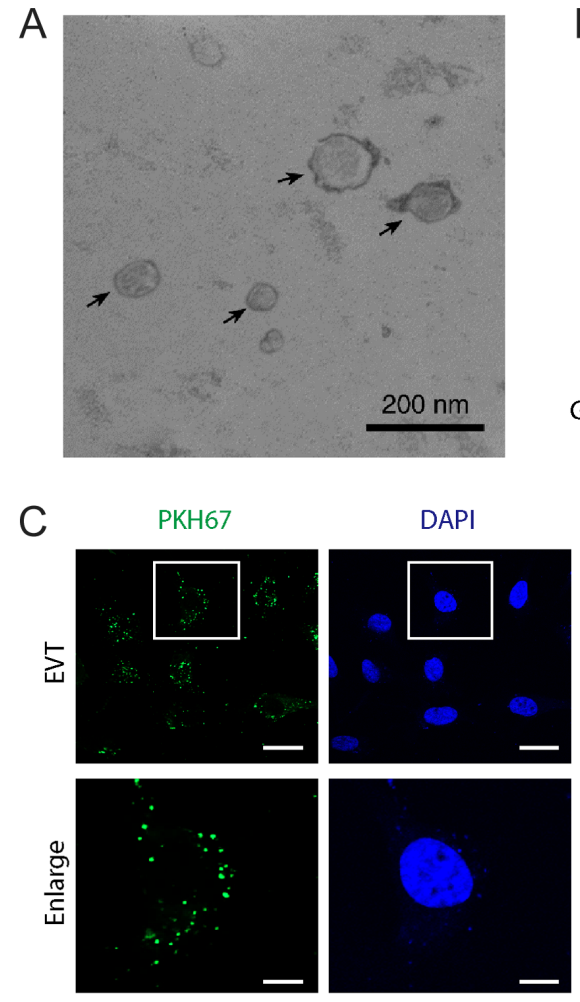

D

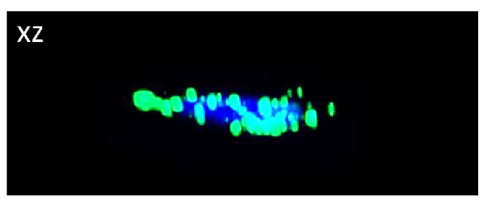

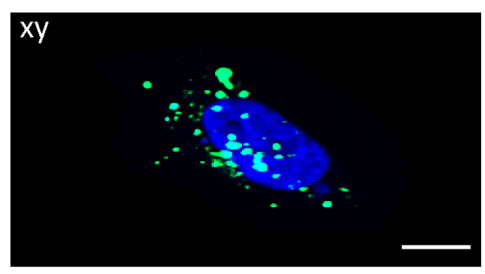

B
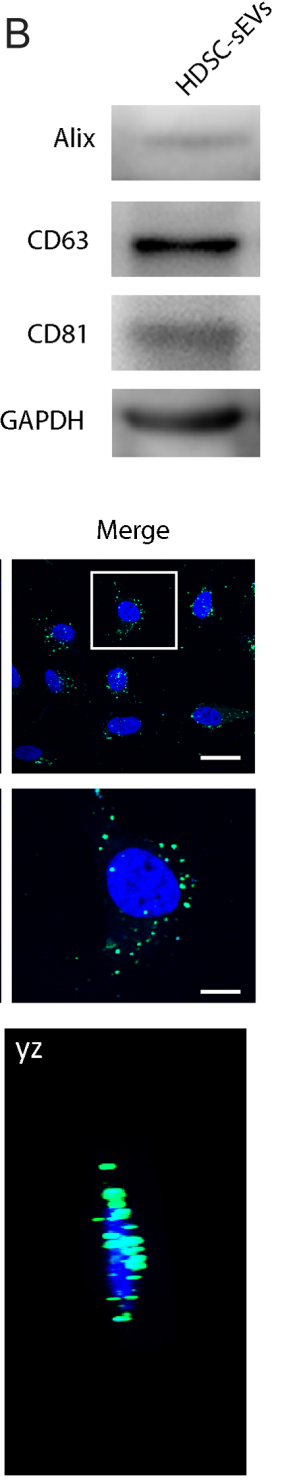

Figure 1 Identification of human decidual stromal cells-derived small extracellular vesicles (HDSC-sEVs) and its uptake by trophoblast cells. (A) Morphology of HDSC-sEVs was confirmed by transmission electron microscopy. Scale bar, $200 \mathrm{~nm}$. (B) Positive markers (Alix, CD63 and CD81) of HDSC-sEVs were detected by Western blotting. (C) Human extravillous trophoblast cells (EVTs), HTR8/SVneo, were pre-treated with PKH67-labeled HDSC-sEVs for $4 \mathrm{~h}$ followed by DAPI (blue) staining for confocal microscopy analysis. Scale bar, 20 $\mu \mathrm{m}$. (D) Representative 3D images (xy/yz/xz) show co-localization of HDSC-sEVs (green) within HTR8 cells. Scale bar, $5 \mu \mathrm{m}$.

SVneo cells (human first-trimester cytotrophoblast cell line usually used in trophoblast biology research) and primary human EVTs. After co-incubation of $\mathrm{PKH}-$ 67-labeled HDSC-sEVs with HTR8/SVneo cells for $4 \mathrm{~h}$, the cells were washed and fixed. Subsequently, the internalization of labeled HDSC-sEVs by HTR-8/ SVneo cells was examined using confocal fluorescence microscopy. Confocal microscopy and z-zack images revealed the uptake of PKH-67-labeled HDSC-
sEVs by HTR-8/SVneo cells (Fig. 1C and D). We also investigated primary HDSC-sEVs uptake kinetics in primary human EVTs where the purity was assessed by positive immunocytochemical staining of trophoblast marker CK7 (Supplementary Fig. 1D), and results show that primary human EVTs can uptake PKH-67-labeled primary-HDSC-sEVs (Supplementary Fig. 1E). These results suggested that the HDSC-sEVs may be involved in the regulation of trophoblast cells function.

\section{HDSC-sEVs induce trophoblast invasion}

To investigate whether HDSC-sEVs affect trophoblast invasion, we used transwell matrigel invasion assay, including matrigel-precoated polycarbonate transwell filters with HTR-8/SVneo cells. The results showed that HDSC-sEVs induced significant HTR8/SVneo cell migration to the undersurface of the transwell filters compared with that without HDSC-sEVs (Fig. 2A and B). HDSC-sEVs significantly increased the invasive ability of HTR-8/SVneo cells at concentrations of $\geq 25 \mu \mathrm{g} / \mathrm{mL}$.

\section{Exposure to HDSC-sEVs increases $\mathrm{N}$-cadherin gene expression and protein levels in trophoblast cells}

To determine the mechanism by which HDSC$\mathrm{sEVs}$ increase the invasiveness of trophoblast cells, we measured the expressions of $\mathrm{N}$-cadherin in the HTR-8/SVneo cells. EMT is a well-recognized process whereby epithelial cells differentiate into a mesenchymal phenotype (Kalluri 2009). N-cadherin is one of the typical markers of EMT and plays a key role in trophoblast invasion (Zhao et al. 2018). Therefore, we assessed the mRNA expression of $\mathrm{N}$-cadherin in trophoblasts by RT-qPCR. We found that the mRNA expression of $\mathrm{N}$-cadherin was significantly increased in HTR-8/SVneo cells treated with HDSC-sEVs (Fig. 2C) or primary EVT cells stimulated by primary HDSC-sEVs after $24 \mathrm{~h}$ (Fig. 2D). Further, Western blotting analysis revealed a significant increase in the protein expression of N-cadherin in HDSC-sEVs-treated HTR8/SVneo cells or primary HDSC-sEVs-treated primary EVT cells after $48 \mathrm{~h}$ stimulation (Fig. 2E and F).

\section{$\mathrm{N}$-cadherin is involved in HDSC-sEVs-induced trophoblast invasion}

To determine whether $\mathrm{N}$-cadherin increase was involved in HDSC-sEVs-induced HTR8/SVneo cells invasion, we used siRNA-mediated knockdown of $\mathrm{N}$-cadherin. Transfection of HTR8/SVneo cells with siRNA targeting $\mathrm{N}$-cadherin for $24 \mathrm{~h}$ suppressed both basal and HDSCsEVs-induced $\mathrm{N}$-cadherin protein levels (Fig. $3 \mathrm{~A}, \mathrm{~B}$ and C). Matrigel-coated transwell invasion assays showed that the decrease of $\mathrm{N}$-cadherin impaired the invasion of both basal and HDSC-sEVs-induced HTR8/SVneo cells (Fig. 3D and E). 
A
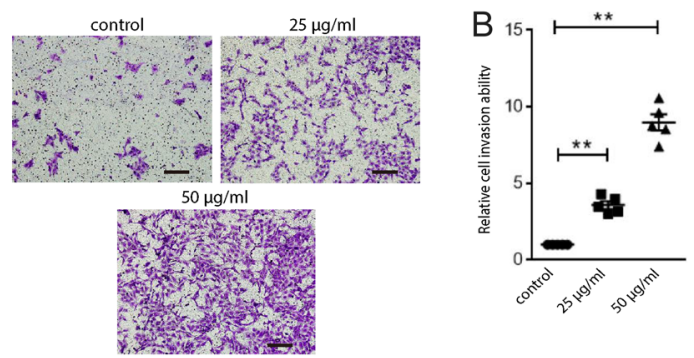

C HTR8/SVneo cells with HDSC-sEVs
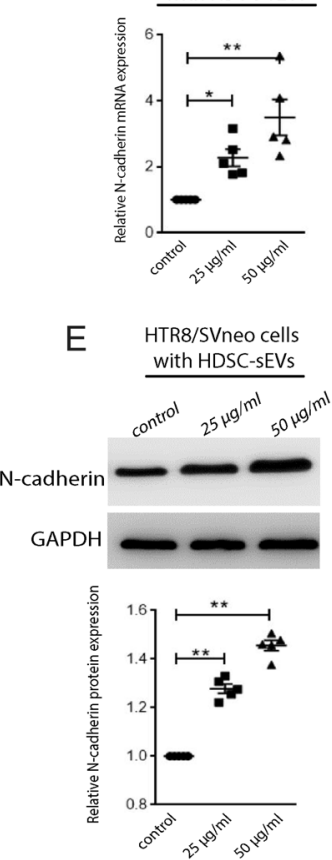

D

HTR8/SVneo cells with HDSC-sEVs

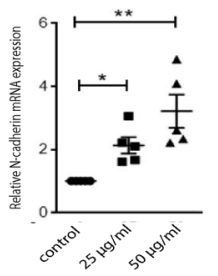

F Primary human EVTs with primary HDSC-sEVs
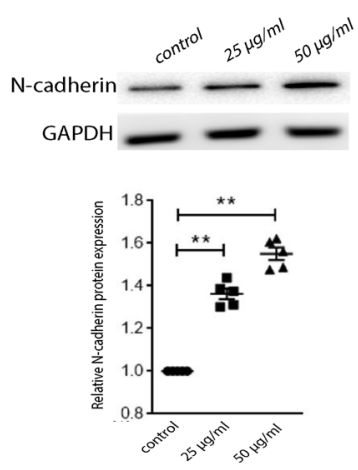

Figure 2 HDSC-sEVs significantly increased the invasiveness of trophoblast cells and induced $\mathrm{N}$-cadherin expression. (A and $\mathrm{B}$ ) Matrigel-coated transwell assay reveals the invasiveness of HTR8/ SVneo with or without HDSC-sEVs $(25 \mu \mathrm{g} / \mathrm{mL}$ and $50 \mu \mathrm{g} / \mathrm{mL})$ pre-treatment. The numbers of invasive cells were quantified in five randomly selected visual fields. and a summary of the statistical analysis was showed. Magnification 100x, scale bar, $100 \mu \mathrm{m}$. (C and D) HTR8/SVneo cells were treated with or without HDSC-sEVs (25 $\mu \mathrm{g} / \mathrm{mL}$ and $50 \mu \mathrm{g} / \mathrm{mL}$ ) for $24 \mathrm{~h}(\mathrm{C})$ or primary EVT cells were treated with or without primary HDSC-sEVs $(25 \mu \mathrm{g} / \mathrm{mL}$ and $50 \mu \mathrm{g} / \mathrm{mL})$ for 24 $\mathrm{h}$ (D) and N-cadherin expression level was evaluated by RT-qPCR with GAPDH as the internal control. (E) Protein level of $\mathrm{N}$-cadherin was detected by Western blotting in HTR8/SVneo cells with or without HDSC-sEVs pre-treatment $(25 \mu \mathrm{g} / \mathrm{mL}$ and $50 \mu \mathrm{g} / \mathrm{mL})$ for $48 \mathrm{~h}$. (F) Protein level of $\mathrm{N}$-cadherin was detected by Western blotting in primary EVT cells with or without primary HDSC-sEVs pre-treatment $(25 \mu \mathrm{g} / \mathrm{mL}$ and $50 \mu \mathrm{g} / \mathrm{mL}$ ) for $48 \mathrm{~h}$. Data were normalized to GAPDH. The results are represented as the means \pm SEM. $n=5$. ${ }^{*} P<0.05$, ${ }^{* *} P<0.01,{ }^{* * *} P<0.001$.

\section{SMAD2/3 signaling is involved in HDSC-sEVs-induced elevation of $\mathrm{N}$-cadherin}

To determine whether HDSC-sEVs induces HTR8/ SVneo cells invasion in a SMAD-dependent manner, we tested SMAD2 and SMAD3 phosphorylation by Western

blotting after the stimulation of HTR8/SVneo cells with $25 \mu \mathrm{g} / \mathrm{mL}$ HDSC-sEVs for 30 or $60 \mathrm{~min}$. HDSC-sEVs significantly increased phosphorylation of SMAD2 and SMAD3 (Fig. 4A). Then we used siRNA to specifically knock down total and phosphorylated SMAD2/3, and the related effects were verified by RT-qPCR and Western blotting (Fig. 4B, C and D). To investigate the combined role of SMAD2 and SMAD3 in HDSC-sEVsinduced increase in $\mathrm{N}$-cadherin, HTR8/SVneo cells were treated with $25 \mu \mathrm{g} / \mathrm{mL}$ HDSC-sEVs after siRNA-mediated depletion of SMAD2 and SMAD3 and the induction of $\mathrm{N}$-cadherin treated with HDSC-sEVs in HTR8/SVneo cells was inhibited by transfected si-Smad2 + si-Smad3. The results showed that knockdown of SMAD2 and SMAD3 significantly abolished the stimulatory effects of HDSC-sEVs on N-cadherin mRNA and protein expressions (Fig. 4E and F).

\section{Discussion}

Here, we demonstrated that human decidual stromal cells could release HDSC-sEVs that induce human trophoblast invasion. In the in vitro study, we induced decidualization of immortal human endometrial stromal cells, isolated primary human decidual stromal cells and collected HDSC-sEVs and primary HDSCsEVs from the culture media. Then, we identified the HDSC-sEVs by morphological analysis and endosomal markers. We demonstrated the uptake of HDSC-sEVs by trophoblast cells in vitro and found that HDSCsEVs could significantly stimulate the invasive potential of trophoblast cells. Our data indicated a significant increase in $\mathrm{N}$-cadherin expression in trophoblast cells stimulated by HDSC-sEVs, and knockdown of $\mathrm{N}$-cadherin by RNAi attenuated this effect. Besides, we observed the upregulation of phosphorylation of SMAD2/3 after HDSC-sEVs treatment. Our findings suggest that HDSC$s E V s$ promoted the invasion of trophoblast cells via the upregulation of $\mathrm{N}$-cadherin through SMAD2/3 signaling pathway. The present study suggested that human decidual stromal cells may participate in trophoblast invasion during embryo implantation and placentation by releasing HDSC-sEVs.

Trophoblast invasion plays an essential role in successful implantation and placentation. In contrast to uncontrolled tumor invasion, the invasion of trophoblast cells is coordinated and controlled by numerous cytokines. Incomplete invasion of trophoblast cells and impaired reconstruction of spiral arteries in decidua result in pregnancy disorders such as PE and fetal growth restriction (Fitzgerald et al. 2008). During embryo implantation and invasion, trophoblasts invade the decidual tissues, which bring them in close contact with the decidual stromal cells. Besides, decidual stromal cells can release many molecules that modulate the trophoblast cells invasion (Sharma et al. 2016). However, the extensive cross-talk between decidual 

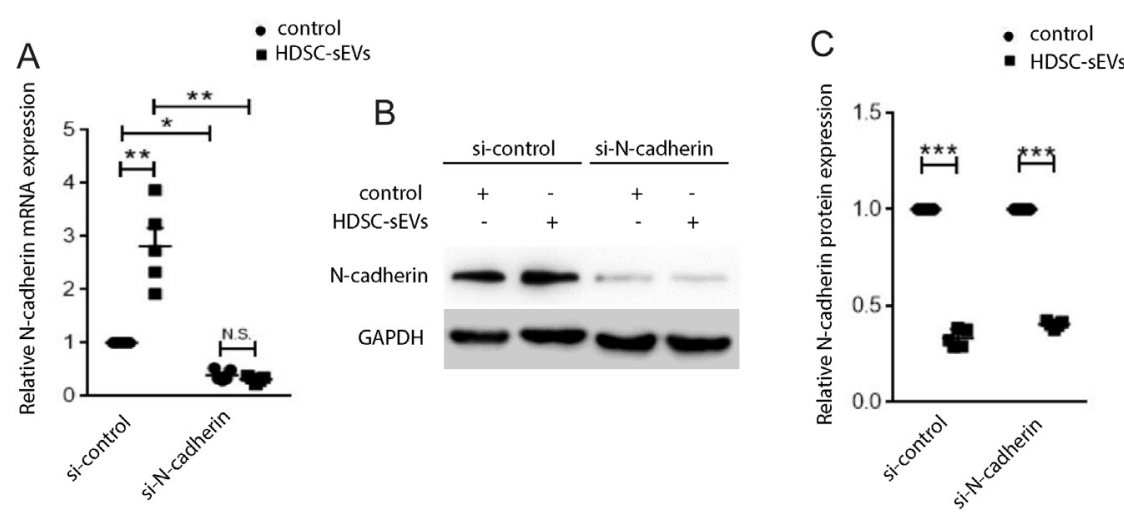

D

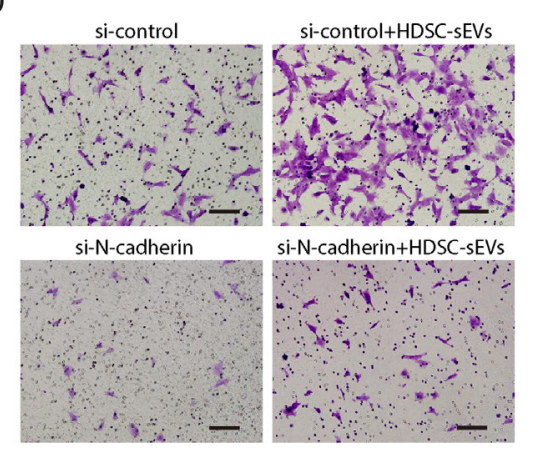

E

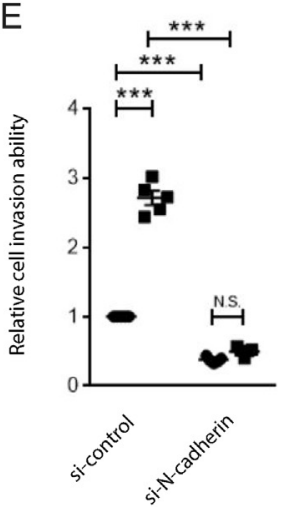

Figure 3 HTR8/SVneo cells invasion was attenuated by siRNA mediated $\mathrm{N}$-cadherin knockdown. (A) HTR8/SVneo cells were transfected with $20 \mathrm{nM}$ nontargeting control siRNA (si-control) or siRNA targeting $\mathrm{N}$-cadherin (si-N-cadherin) for $24 \mathrm{~h}$ before HDSC-sEVs treatment. After $24 \mathrm{~h}$ treatment with or without HDSC-sEVs, mRNA level of $\mathrm{N}$-cadherin was detected by RT-qPCR using $\mathrm{GAPDH}$ as the internal control. (B and C) After $48 \mathrm{~h}$ treatment with or without HDSC-sEVs, protein levels of $\mathrm{N}$-cadherin in HTR8/SVneo cells were detected by Western blotting, and data were normalized to GAPDH. (D and E) The effects of $\mathrm{N}$-cadherin knockdown on HDSC-sEVs-induced invasion of HTR8/SVneo cells were determined by transwell assay. Representative images were shown. Magnification 100x, scale bar, $100 \mu \mathrm{m}$. The results are represented as the means \pm SEM. $n=5$. ${ }^{*} P<0.05,{ }^{* *} P<0.01,{ }^{* * *} P<0.001$. stromal cells and trophoblast cells, especially the mechanism by which decidual stromal cells regulate trophoblast invasion, is not completely understood. Our study suggested human decidual stromal cells may regulate trophoblast invasion by releasing sEVs.

EVs are vesicles released by most cells including exosomes, microvesicles and apoptotic body. sEVs represent a particular subtype of extracellular vesicles with the diameter of $40-300 \mathrm{~nm}$, and sEVs contain various signaling molecules, including mRNA, microRNA, LncRNA and proteins (Raposo \& Stoorvogel 2013, Théry et al. 2018). sEVs play an important role in cell-to-cell crosstalk and are involved in many physical and pathological events including pregnancy (Homer et al. 2017). During normal pregnancy, the plasma concentration of sEVs increases significantly compared with non-pregnant women (Sabapatha et al. 2006). sEVs isolated from pregnant women can reportedly promote endothelial cell migration, indicating that $s E V s$ in plasma of normal pregnant women may have bioactive functions (Salomon et al. 2014). Besides, the total concentration of $\mathrm{sEVs}$ in preeclamptic women was reported to be significantly higher than that of normal pregnant women, indicating that $\mathrm{sEV}$ s may contribute to the occurrence of PE (Pillay et al. 2017). In contrast to many studies focused on SEVs derived from syncytiotrophoblast cells, few studies investigated the role of HDSC-sEVs during embryo implantation and placentation. In this study, we collected HDSC-sEVs and performed transwell chamber assay to investigate the effect of HDSC-sEVs on invasion capacity of trophoblast cell line HTR8/SVneo cells. It turned out that HDSC-sEVs could significantly promote the invasiveness of HTR8/SVneo cells, which suggested that HDSC-sEVs may play an important role in trophoblast cells invasion. We further investigated the mechanism of HDSC-sEVs in regulating trophoblast invasion, and elevated expression level of $\mathrm{N}$-cadherin, hallmark of EMT, was detected in trophoblast cells. EMT is characterized by a decrease in the expression of epithelial markers, such as E-cadherin, and increase in the expressions of mesenchymal markers, such as $\mathrm{N}$-cadherin and vimentin (Christiansen \& Rajasekaran 2006, Xu et al. 2009). Trophoblast invasion is closely associated with EMT, and abnormal EMT may be involved in the pathogenesis of pregnancy-related disorders (DaSilva-Arnold et al. 2018). N-cadherin is one of the typical markers of EMT, and many studies pointed out that $\mathrm{N}$-cadherin played a key role in trophoblast invasion (Li et al. 2014, Zhao et al. 2018). Our study focused on the role of $\mathrm{N}$-cadherin in HDSC-sEVs promoting trophoblast invasion, and we found that HDSC-sEVs induced $\mathrm{N}$-cadherin expression and invasiveness of trophoblast cells could be blocked when $\mathrm{N}$-cadherin was knocked down. In our subsequent experiments, we found that HDSC-sEVs could upregulate $\mathrm{N}$-cadherin expression through SMAD2/3 signaling. SMAD2/3 are important transcription factors in TGF- $\beta$ superfamily signaling pathway. This signaling pathway is involved 

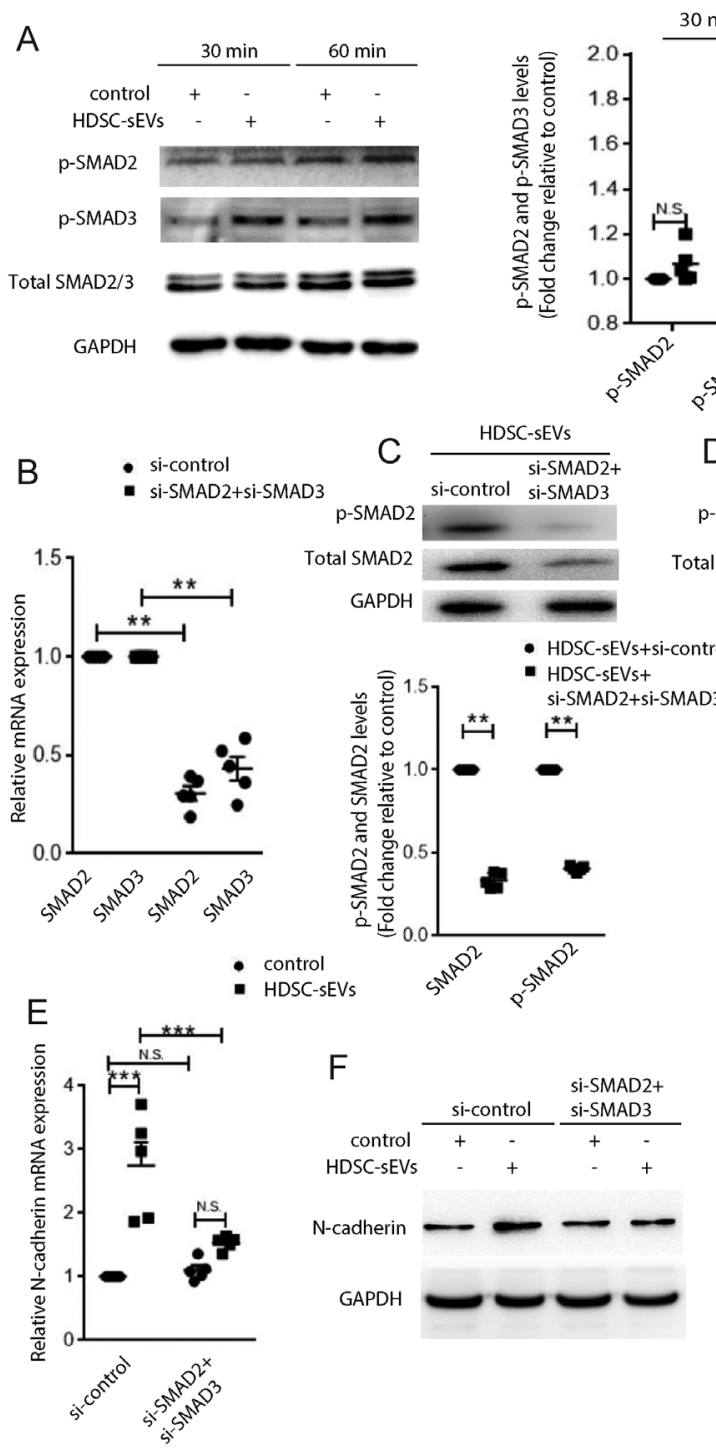
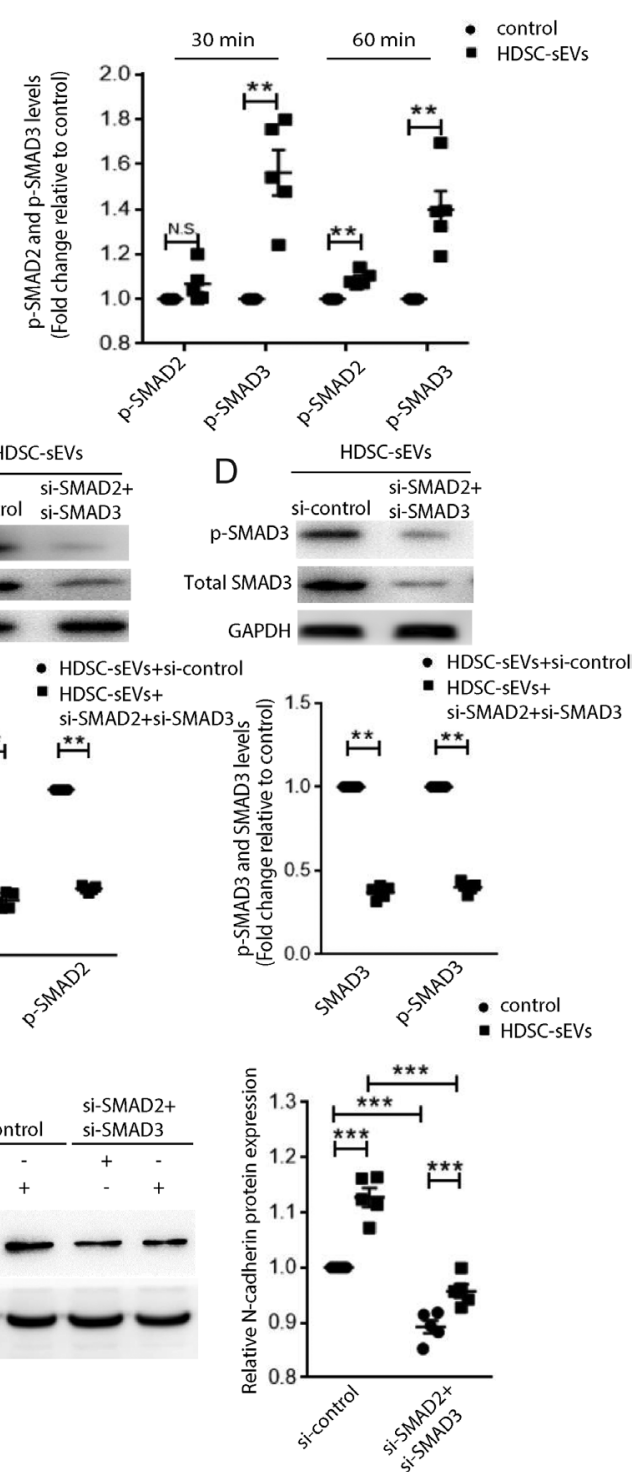

Figure $4 \mathrm{SMAD} 2 / 3$ signaling mediates the HDSC-EVs-induced upregulation of $\mathrm{N}$-cadherin. (A) HTR8/SVneo cells were treated with or without $25 \mu \mathrm{g} / \mathrm{mL}$ HDSC-sEVs for different lengths of time (30 or $60 \mathrm{~min}$ ). Phosphorylated SMAD2 and SMAD3 (p-SMAD2 and p-SMAD3) levels were detected by Western blotting with corresponding phospho-specific antibodies. (B) HTR8/SVneo cells were transfected with 20 $\mathrm{nM}$ scrambled negative control siRNA (si-control) or siRNA targeting SMAD2 + SMAD3 (si-SMAD2 + 3) for $24 \mathrm{~h}$, and mRNA levels of SMAD2/SMAD3 were tested by RT-qPCR using GAPDH as reference. (C) SMAD2 protein and $\mathrm{p}-\mathrm{SMAD} 2$ protein expressions were significantly reduced in HTR8/SVneo cells transfected with si-SMAD2 + 3, compared to scramble transfected cells. (D) SMAD3 protein and p-SMAD3 protein expression were significantly reduced in HTR8/SVneo cells transfected with si-SMAD2 +3, compared to scramble transfected cells. (E) After being transfected with si-SMAD2 +3 or si-control for $24 \mathrm{~h}, \mathrm{HTR} 8 / \mathrm{SV}$ neo cells were treated with or without $25 \mu \mathrm{g} / \mathrm{mL}$ HDSC-sEVs. After $24 \mathrm{~h}$, $\mathrm{N}$-cadherin mRNA levels were detected by RT-qPCR using GAPDH as reference ( $F)$. After $48 \mathrm{~h}$, protein levels of $\mathrm{N}$-cadherin in HTR8/ SVneo cells were detected by Western blotting and the data were normalized to GAPDH (E and $\mathrm{F}$ ). The results are represented as the means \pm SEM. $n=5 .{ }^{*} P<0.05,{ }^{* *} P<0.01$, $* * * P<0.001$. in the modulation of hormone production and cancer progression (Holtzhausen et al. 2014).

The $\mathrm{sEV}$ s contents are diverse and are regulated by the microenvironment (Homer et al. 2017). Menstrual cycle hormones can modulate the cargoes of endometrial epithelial-derived sEVs. Previous study demonstrates that the molecular contents are different between estrogen-treated endometrial epithelial-derived exosomes and estrogen plus progesterone-treated endometrial epithelial-derived exosomes, and many proteins are not reported before in exosomes database (Greening et al. 2016). During embryo implantation and placentation, significant changes occur within the uterine microenvironment, such as changes in hormone levels, and it will be interesting to further explore the unique molecules in HDSC-sEVs cargo compared with related database (Greening et al. 2016). Proteomic analysis should be performed to identify the specific molecules of HDSC-sEVs that regulate the SMAD2/3-N-cadherin pathway to modulate the invasiveness of trophoblast cells. Besides, more experiments should be performed on primary human trophoblast to further identify the role of HDSC-sEVs on trophoblast bioactivities during implantation and placentation.

In summary, our study demonstrated that the HDSCsEVs could induce the invasion of trophoblast cells through the SMAD2/3-N-cadherin signaling pathway. Decidua could modulate the function of trophoblast cells by releasing HDSC-sEVs. Our study provides new insights into the interaction of trophoblasts and decidual stromal cells. It may help us to further understand the mechanisms of the occurrence of pregnancy-related disorders. Further studies should be performed to investigate the diverse changes in HDSC-sEVs under different uterine milieu and their involvement in many pregnancy-related disorders. The particular contents of HDSC-sEVs in different uterine microenvironments should be revealed to demonstrate 
the signaling pathways that regulate the bioactivities of trophoblast cells.

\section{Supplementary materials}

This is linked to the online version of the paper at https://doi.org/10.1530/REP-18-0616.

\section{Declaration of interest}

The authors declare that there is no conflict of interest that could be perceived as prejudicing the impartiality of the research reported.

\section{Funding}

This work was supported by the National Key Research and Development Program of China (2018YFC1004400, 2016YFC1000205), National Natural Science Foundation of China (81471464, 81771609) and the Natural Science Foundation of Guangdong Province (2017A030313584).

\section{Author contribution statement}

$M L$ and $X C$ conceived the ideas and designed the experiments. $Y X W, X J Y$ and $F S$ performed the experiments. Q X C, L $\mathrm{PH}$ and $\mathrm{Y} X \mathrm{~L}$ provided the patient samples for scientific investigation. S J J H Y H analyzed the data. M L, R H wrote the paper. S Q, Y H Y led the project, interpreted the data and drafted critical revisions.

\section{Acknowledgments}

The authors thank Prof. Hai-Bing Wang, PhD, Xiamen University, for generously providing human endometrial stromal cell line.

\section{References}

Brosens I, Robertson WB \& Dixon HG 1967 The physiological response of the vessels of the placental bed to normal pregnancy. Journal of Pathology and Bacteriology 93 569-579. (https://doi.org/10.1002/ path.1700930218)

Carson DD, Bagchi I, Dey SK, Enders AC, Fazleabas AT, Lessey BA \& Yoshinaga K 2000 Embryo implantation. Developmental Biology 223 217-237. (https://doi.org/10.1006/dbio.2000.9767)

Christiansen JJ \& Rajasekaran AK 2006 Reassessing epithelial to mesenchymal transition as a prerequisite for carcinoma invasion and metastasis. Cancer Research 66 8319-8326. (https://doi. org/10.1158/0008-5472.CAN-06-0410)

DaSilva-Arnold SC, Zamudio S, Al-Khan A, Alvarez-Perez J, Mannion C, Koenig C, Luke D, Perez AM, Petroff M, Alvarez M et al. 2018 Human trophoblast epithelial-mesenchymal transition in abnormally invasive placenta. Biology of Reproduction 99 409-421. (https://doi.org/10.1093/ biolre/ioy042)

Dey SK, Lim H, Das SK, Reese J, Paria BC, Daikoku T \& Wang H 2004 Molecular cues to implantation. Endocrine Reviews 25 341-373. (https:// doi.org/10.1210/er.2003-0020)

Fitzgerald JS, Poehlmann TG, Schleussner E \& Markert UR 2008 Trophoblast invasion: the role of intracellular cytokine signalling via signal transducer and activator of transcription 3 (STAT3). Human
Reproduction Update 14 335-344. (https://doi.org/10.1093/humupd/ dmn010)

Gellersen B \& Brosens J 2014 Cyclic decidualization of the human endometrium in reproductive health and failure. Endocrine Reviews $\mathbf{3 5}$ 851-905. (https://doi.org/10.1210/er.2014-1045)

Godbole G, Suman P, Gupta SK \& Modi D 2011 Decidualized endometrial stromal cell derived factors promote trophoblast invasion. Fertility and Sterility 95 1278-1283. (https://doi.org/10.1016/j.fertnstert.2010.09.045)

Gopal SK, Greening DW, Rai A, Chen M, Xu R, Shafiq A, Mathias RA, Zhu HJ \& Simpson RJ 2017 Extracellular vesicles: their role in cancer biology and epithelial-mesenchymal transition. Biochemical Journal $\mathbf{4 7 4}$ 21-45. (https://doi.org/10.1042/BCJ20160006)

Graham CH, Hawley TS, Hawley RG, MacDougall JR, Kerbel RS, Khoo N \& Lala PK 1993 Establishment and characterization of first trimester human trophoblast cells with extended lifespan. Experimental Cell Research 206 204-211. (https://doi.org/10.1006/excr.1993.1139)

Greening DW, Nguyen HP, Elgass K, Simpson RJ \& Salamonsen LA 2016 Human endometrial exosomes contain hormone-specific cargo modulating trophoblast adhesive capacity: insights into endometrialembryo interactions. Biology of Reproduction 94 38. (https://doi. org/10.1095/biolreprod.115.134890)

Hansen SM, Berezin V \& Bock E 2008 Signaling mechanisms of neurite outgrowth induced by the cell adhesion molecules NCAM and N-cadherin. Cellular and Molecular Life Sciences 65 3809-3821. (https:// doi.org/10.1007/s00018-008-8290-0)

Holtzhausen A, Golzio C, How T, Lee YH, Schiemann WP, Katsanis N \& Blobe GC 2014 Novel bone morphogenetic protein signaling through Smad2 and Smad3 to regulate cancer progression and development. FASEB Journal 28 1248-1267. (https://doi.org/10.1096/fj.13-239178)

Homer H, Rice GE \& Salomon C 2017 Review: embryo- and endometriumderived exosomes and their potential role in assisted reproductive treatments-liquid biopsies for endometrial receptivity. Placenta $\mathbf{5 4}$ 89-94. (https://doi.org/10.1016/j.placenta.2016.12.011)

James-Allan LB, Whitley GS, Leslie K, Wallace AE \& Cartwright JE 2018 Decidual cell regulation of trophoblast is altered in pregnancies at risk of pre-eclampsia. Journal of Molecular Endocrinology 60 239-246. (https:// doi.org/10.1530/JME-17-0243)

Johnson GA, Bazer FW, Jaeger LA, Ka H, Garlow JE, Pfarrer C, Spencer TE \& Burghardt RC 2001 Muc-1, integrin, and osteopontin expression during the implantation cascade in sheep. Biology of Reproduction $\mathbf{6 5}$ 820-828. (https://doi.org/10.1095/biolreprod65.3.820)

Kalluri R 2009 EMT: when epithelial cells decide to become mesenchymallike cells. Journal of Clinical Investigation 119 1417-1419. (https://doi. org/10.1172/JCI39675)

Krikun G, Mor G, Alvero A, Guller S, Schatz F, Sapi E, Rahman M, Caze R, Qumsiyeh M \& Lockwood CJ 2004 A novel immortalized human endometrial stromal cell line with normal progestational response. Endocrinology 145 2291-2296. (https://doi.org/10.1210/en.2003-1606)

Li MQ, Hou XF, Shao J, Tang CL \& Li DJ 2010 The DSCs-expressed CD82 controls the invasiveness of trophoblast cells via integrinbeta1/ MAPK/MAPK3/1 signaling pathway in human first-trimester pregnancy. Biology of Reproduction 82 968-979. (https://doi.org/10.1095/ biolreprod.109.080739)

Li Y, Klausen C, Cheng JC, Zhu H \& Leung PCK 2014 Activin A, B, and AB increase human trophoblast cell invasion by up-regulating $\mathrm{N}$-cadherin. Journal of Clinical Endocrinology and Metabolism 99 E2216-E2225. (https://doi.org/10.1210/jc.2014-2118)

Li X, Wang Y, Wei P, Shi D, Wen S, Wu F, Liu L, Ye N \& Zhou H 2018 Bisphenol A affects trophoblast invasion by inhibiting CXCL8 expression in decidual stromal cells. Molecular and Cellular Endocrinology 470 38-47. (https://doi.org/10.1016/j.mce.2017.07.016)

Maas SLN, Breakefield XO \& Weaver AM 2017 Extracellular vesicles: unique intercellular delivery vehicles. Trends in Cell Biology 27 172-188. (https://doi.org/10.1016/j.tcb.2016.11.003)

Mrozik KM, Blaschuk OW, Cheong CM, Zannettino ACW \& Vandyke K 2018 $\mathrm{N}$-cadherin in cancer metastasis, its emerging role in haematological malignancies and potential as a therapeutic target in cancer. BMC Cancer 18 939. (https://doi.org/10.1186/s12885-018-4845-0)

Pijnenborg R, Anthony J, Davey DA, Rees A, Tiltman A, Vercruysse L \& van Assche A 1991 Placental bed spiral arteries in the hypertensive disorders of pregnancy. British Journal of Obstetrics and Gynaecology 98 648-655. (https://doi.org/10.1111/j.1471-0528.1991.tb13450.x) 
Pillay P, Moodley K, Moodley J \& Mackraj I 2017 Placenta-derived exosomes: potential biomarkers of preeclampsia. International Journal of Nanomedicine 12 8009-8023. (https://doi.org/10.2147/IJN.S142732)

Raposo G \& Stoorvogel W 2013 Extracellular vesicles: exosomes, microvesicles, and friends. Journal of Cell Biology $200373-383$. (https:// doi.org/10.1083/jcb.201211138)

Red-Horse K, Zhou Y, Genbacev O, Prakobphol A, Foulk R, McMaster M \& Fisher SJ 2004 Trophoblast differentiation during embryo implantation and formation of the maternal-fetal interface. Journal of Clinical Investigation 114 744-754. (https://doi.org/10.1172/JCI22991)

Sabapatha A, Gercel-Taylor C \& Taylor DD 2006 Specific isolation of placenta-derived exosomes from the circulation of pregnant women and their immunoregulatory consequences. American Journal of Reproductive Immunology 56 345-355. (https://doi.org/10.1111/j.16000897.2006.00435.x)

Salomon C, Torres MJ, Kobayashi M, Scholz-Romero K, Sobrevia L, Dobierzewska A, Illanes SE, Mitchell MD \& Rice GE 2014 A gestational profile of placental exosomes in maternal plasma and their effects on endothelial cell migration. PLoS ONE 9 e98667. (https://doi.org/10.1371/ journal.pone.0098667)

Sankaralingam S, Arenas IA, Lalu MM \& Davidge ST 2006 Preeclampsia: current understanding of the molecular basis of vascular dysfunction. Expert Reviews in Molecular Medicine 8 1-20. (https://doi.org/10.1017/ S1462399406010465)

Sharma S, Godbole G \& Modi D 2016 Decidual control of trophoblast invasion. American Journal of Reproductive Immunology 75 341-350. (https://doi.org/10.1111/aji.12466)

Szwarc MM, Hai L, Gibbons WE, Peavey MC, White LD, Mo Q, Lonard DM, Kommagani R, Lanz RB, DeMayo FJ et al. 2018 Human endometrial stromal cell decidualization requires transcriptional reprogramming by PLZF. Biology of Reproduction 98 15-27. (https://doi.org/10.1093/ biolre/iox161)

Tannetta D, Collett G, Vatish M, Redman C \& Sargent I 2017 Syncytiotrophoblast extracellular vesicles - circulating biopsies reflecting placental health. Placenta 52 134-138. (https://doi.org/10.1016/j. placenta.2016.11.008)

Théry C, Witwer KW, Aikawa E, Alcaraz MJ, Anderson JD, Andriantsitohaina R, Antoniou A, Arab T, Archer F, Atkin-Smith GK et al. 2018 Minimal information for studies of extracellular vesicles 2018 (MISEV2018): a position statement of the International Society for Extracellular Vesicles and update of the MISEV2014 guidelines. Journal of Extracellular Vesicles 7 1535750. (https://doi.org/10.1080/20013078. 2018.1535750)

Wu D, Lu P, Mi X \& Miao J 2018 Exosomal miR-214 from endometrial stromal cells inhibits endometriosis fibrosis. Molecular Human Reproduction 24 357-365. (https://doi.org/10.1093/molehr/gay019)

Xu J, Lamouille S \& Derynck R 2009 TGF-beta-induced epithelial to mesenchymal transition. Cell Research 19 156-172. (https://doi. org/10.1038/cr.2009.5)

Yan W, Wu X, Zhou W, Fong MY, Cao M, Liu J, Liu X, Chen CH, Fadare O, Pizzo DP et al. 2018 Cancer-cell-secreted exosomal miR-105 promotes tumour growth through the MYC-dependent metabolic reprogramming of stromal cells. Nature Cell Biology 20 597-609. (https://doi.org/10.1038/ s41556-018-0083-6)

Zhao HJ, Chang HM, Zhu H, Klausen C, Li Y \& Leung PCK 2018 Bone morphogenetic protein 2 promotes human trophoblast cell invasion by inducing activin A production. Endocrinology 159 2815-2825. (https:// doi.org/10.1210/en.2018-00301)

Received 7 December 2018

First decision 16 January 2019

Revised manuscript received 19 November 2019

Accepted 29 November 2019 